\title{
REMITTING SERONEGATIVE SYMMETRICAL SYNOVITIS WITHPITTING EDEMA (RS3PE) SYNDROME ASSOCIATED WITH ANTI-GAD ANTIBODY- RELATED LIMBIC ENCEPHALITIS
}

\author{
Jean Michell Correia Monteiro ${ }^{1}{ }^{\star}$, Mateus Cavarzan Lopes ${ }^{1}$, Heitor Furlan Giordano ${ }^{1}$, Diogo Souza Domiciano ${ }^{1}$, Samuel \\ Katsuyuki Shinjo ${ }^{1}$ \\ 1. Hospital das Clinicas HCFMUSP, Faculdade de Medicina, Universidade de São Paulo, São Paulo (SP), Brazil. \\ *Corresponding author: jk_michell@hotmail.com
}

\section{BACKGROUND}

Remitting seronegative symmetrical synovitis with pitting oedema (RS3PE) syndrome is a rare form of polyarthritis that affects elderly people, and it is characterized by symmetrical involvement of small joints, marked pitting edema on the dorsum of the hands and feet, sudden inflammatory onset, absence of rheumatoid factor, and rapid response to glucocorticoids. It may be paraneoplastic manifestation, but it can also be the initial presentation of rheumatic diseases. There is no case of RS3PE associated with glutamic acid decarboxylase (GAD)-related neurological syndromes such as limbic encephalitis (LE) and cerebellar ataxia (CA) reported in the literature.

\section{CASE REPORT}

A 62-year-old woman with a 7-year history of anti-GAD syndrome (GADS) consisting of LE, CA and epileptic seizures was admitted with ataxic gait started two weeks earlier. Also, she reported intermittent arthritis in ankles and wrists, unintentional weight loss and intermittent fever, without pain in shoulder and pelvic girdle. She had been in remission of the neurological disease for the last five years using azathioprine $3 \mathrm{mg} / \mathrm{kg} /$ day. Her medical history was remarkable for cervical cancer treated 7 years before GADS diagnosis and pernicious anemia. On examination, she had drunk pattern gait, synovitis in wrists and ankles and remarkable pitting oedema on the dorsum of the hands and feet. Ultrasonography confirmed synovitis in radiocarpal, midcarpal and tibiotalar joints without tenosynovitis or joint effusion. Laboratory tests showed a high ESR $(81 \mathrm{~mm} / \mathrm{h})$ and high CRP $(100 \mathrm{mg} / \mathrm{L})$. Blood cultures, infectious serologies and autoantibodies were negative, including rheumatoid factor, anti-CCP and ANA. Brain MRI did not show new findings and cerebral fluid analysis was unremarkable. Due to suspected GADS relapse, neurological team started intravenous methylprednisolone $1 \mathrm{~g} /$ day for 5 days. After 1 week, there was significant improvement of neurological and articular symptoms. The PET-CT scan showed a right axillary lymph node uptake, but mammography and breast ultrasound were normal. Paraneoplastic RS3PE syndrome was suspected, but lymph node histology demonstrated reactive lymphadenitis. Thus, she was referred for outpatient follow-up using methotrexate $15 \mathrm{mg} /$ week.

\section{CONCLUSION}

To the best of our knowledge, this is the first patient with RS3PE associated with GADS described in medical literature. This case extends previously reported findings of association between GADS and autoimmune disorders such as diabetes mellitus type 1, thyroiditis, and pernicious anemia. In addition, careful attention should be paid to this patient regarding cancer screening at the follow-up, given that, GADS can also be paraneoplastic manifestation likewise RS3PE, although less frequently.

\section{KEYWORDS}

RS3PE, limbic encephalitis, paraneoplastic syndrome. 